Reprod. Nutr. Dévelop., 1988, 28 (6 B), 1587-1598.

\title{
Rôle du placenta dans le maintien de l'allogreffe fétale
}

\author{
G. CHAOUAT, Elisabeth MENU, P. BUSTANY, Charlyne REBUT-BONNETON, \\ Julia SZEKERES-BARTHO $\left({ }^{* *}\right)$, T. G. WEGMANN $\left({ }^{*}\right)$
}

Unité 262 INSERM, Clinique Universitaire Baudelocque, 123, bd de Port Royal, 75674 Paris Cedex 14, France.

(*) Université d'Alberta, MRC Group in Immunology, Edmonton. Alberta, Canada T6G 2 H7.

(**) Pecs University, Hungary.

Summary. Role of the placenta in maintaining the foetal allograft.

Present knowledge of the alloantigenic status of the placenta, which makes it a natural semi-allogeneic allograft is briefly surveyed. The systemic immunoregulatory mechanisms operating during normal allopregnancy which are not a prerequisite for a successful pregnancy are recalled. The placenta - dependent local mechanisms, e.g. trophoblast dependent decidual suppressor cells, factor mediated and factor independent resistance to cell mediated lysis, are surveyed as well as some of the mechanisms of action of trophoblast regulatory factors, namely suppressor cell induction and inhibition of IL-2 dependent cell growth/activation. The main feature of the CBA $\times \mathrm{DBA} / 2$ model of spontaneous abortions in mice, and its prevention by anti Balb/c leukocyte immunisation are described. It was shown that anti-T cell depletion prevents the anti-abortive effects of immunisation. Such a treatment is also able to restore normal placental weight in auto-immune MRL Ipr mice, which are known to display excess seric CSF beta-like activity (CSFs being in vitro efficient growth factors for trophoblasts). Transfer of such cells into the MHC compatible $\mathrm{CBA} / J$ prevents resorbtions upon a subsequent mating with DBA/2. Thus, direct effects of CSFs beta 1 and beta 2 as anti abortifacient (IL3 and GM CSF) are described together with the abortificacient effects of TNFs and NKs activators.

\section{Introduction.}

II existe maintenant des preuves indiscutables, fournies par les travaux récents du groupe de Judith Head (Drake et al., 1988a), utilisant des techniques de biologie moléculaire, chez la souris, et par sérologie classique, chez le rat, du groupe de Billington (Billington et Burrows, 1986), que le spongiotrophoblaste de rongeurs exprime des alloantigènes d'histocompatibilité majeurs ( $\mathrm{MHC}$ ) polymorphes de classe I (K, D, L). La transcription débute dès le jour 7,5, et l'expression optimale à la surface des cellules placentaires se fait au jour 10,5. L'utérus est rempli de cellules classe II positives, ayant la capacité, même dans la déciduale, de présenter des alloantigènes au système immunitaire maternel (Matthews et Searle, 1988). Chez l'homme, la situation est la même pour la déciduale (Bulmer et Ritson, 1985; Dorman et Searle, 1988); le placenta 
n'exprime aucun antigène HLA sur le syncytiotrophoblaste, mais il y a des cellules exprimant des antigènes classe I sur les membranes choriales (Hunt et Fishback, 1988). Le placenta devrait donc être rejeté. II n'en est rien, et, au contraire, une différence antigénique mère-père est favorable au développement fetal, mesuré par le poids de l'unité feto-placentaire. Les antigènes dits «mineurs » semblent jouer à cet égard un rôle important (Hamilton et Hamilton, 1987b). Les mécanismes de survie de l'unité feto-placentaire sont très dépendants du placenta : induction au cours d'une gestation d'une réponse anticorps à prédominance non cytotoxique (Voisin et Chaouat, 1974 ; Bell et Billington, 1983). Cette prédominance confère à ces anticorps un rôle "facilitant " (Chaouat et al., 1979). De fait, à l'opposé d'une immunisation normale ou, par du tissu fetal, qui induit une réponse à forte composante lgG2 (cytotoxique), l'immunisation par du tissu placentaire (Bell et Billington, 1983; Tartakowsky et al., 1981), ou par du tissu adulte plus des extraits placentaires (Duc et al., 1985) se traduit par une réponse anticorps facilitante. De même, l'immunisation dans de telles conditions entraîne I'apparition de cellules T suppressives capables de transférer in vivo une hyporéponse aux antigènes du père (Chaouat et al., 1979; Smith et Powell, 1977 ; Chaouat et al., 1980), qui s'accompagne d'ailleurs de modifications caractéristiques des sous-populations thymiques (Chaouat et al., 1982). Mais l'attention s'est portée sur les mécanismes régulateurs locaux, parce que les souris dans leur très grande majorité ne font pas d'anticorps anti-paternels (Bell et Billington, 1983), et surtout parce que les mécanismes systémiques peuvent être éliminés sans conséquence néfaste pour le fœtus (Mattson, Mattson et Sulila, 1985 ; Rodger, 1985 ; Mattson et Holmdal, 1987). On peut même générer in vivo des effecteurs cytotoxiques cellulaires maternels antipaternels sans compromettre une gestation (Mitchison, 1953 ; Lanman et al., 1982 ; Wegmann et al., 1979 ; Chaouat et Monnot, 1984).

L'un des mécanismes locaux est l'existence de cellules suppressives dans la déciduale. L'une au moins des populations suppressives de la déciduale est sous contrôle placentaire et peut être hormonal (Clark, 1985; Clark et al., 1986; Slapsys et al., 1986).

Toutefois, le placenta normal est soit peu sensible à la lyse par effecteurs cellulaires si on prépare des suspensions de cellules trophoblastiques par la trypsine (Smith, 1983), soit pas du tout sensible (préparation à la collagénase), que ce soit par cellules NKs (Kolb et Chaouat, 1984) ou CTLs (Chaouat et Kolb, 1985 ; Zuckerman et Head, 1987 ; Croy et Rossant, 1987). Cette résistance est en partie intrinsèque (Croy et Rossant, 1987 ; Zuckerman et Head, 1987 ; Chaouat, 1988), en partie due à une inhibition non compétitive (Clark et Chaouat, 1986), qui est due à différents facteurs solubles. (Pour revue, voir Chaouat, 1987 ; Menu et Chaouat, 1988.) Toutefois, le placenta serait un peu sensible à la lyse par LAKCs hyper activés (on notera le parallèle avec certaines tumeurs malignes) (Drake et Head, 1988). Chez l'homme, nous avons commencé l'étude de tels facteurs dans des surnageants de culture de placenta et de choriocarcinomes. Ainsi, l'incubation du clone JF-1 (travail réalisé avec A. Bensussan et collaborateurs), qui est un clone à « double » activité, NK like et CTL spécifique, se traduit 
par une inhibition de ses 2 types de cytotoxicité. Cette inhibition est réversible par excès d'interleukine 2 (tabl. $1 \mathrm{a}$ et $1 \mathrm{~b}$ ).

TABLEAU 1a

Inhibition par le surnageant de placenta humain de la lyse par JF-1.

\begin{tabular}{lcc}
\hline Cellules incubées en $2 \mathrm{~h}$ en : Rapport E/T & $\%$ Lyse & Inhibition. \\
\hline Milieu de culture (Contrôle) $(5: 1)$ & $35,06 \%$ & - \\
& $\pm 0,98 \%$ & \\
Surnageant au $1: 10$ & $17,47 \%$ & $51<0,01$ \\
& $\pm 1,95$ & $86<0,01$ \\
Surnageant au $1: 30$ & $5,4 \%$ & $22<0,01$ \\
& $\pm 1,46$ & \\
Surnageant au $1: 90$ & $27,99 \%$ & $\pm 0,24$ \\
\hline
\end{tabular}

Les cellules du clone JF-1 sont ici testées après incubation de 2 h sur la cible NK classique K 562 marquée au Chrome 51. Le test est un test CRT classique de $4 \mathrm{~h}$.

TABLEAU $1 \mathrm{~b}$

Inhibition par le surnageant placentaire de la lyse médiée par le clone JF- 1 après expansion de celui-ci en interleukine $2(\mathrm{~L}-2)$ et levée partielle de cette inhibition par rajout d'IL2 en excès au début du test de lyse.

\begin{tabular}{|c|c|c|c|c|c|c|}
\hline Cible & Rapport E : T & Pousse en & Milieu du test & cpms & $\%$ lyse & $\mathrm{p}$ \\
\hline $\begin{array}{c}\text { K } 562 \\
\text { (Cible NK) }\end{array}$ & $\begin{array}{c}0: \text { (Spont) } \\
0 \\
30: 1 \\
30: 1 \\
30: 1\end{array}$ & $\begin{array}{c}- \\
\overline{I L-2} \\
\mathrm{~S} / \mathrm{N} 1: 3 \\
\mathrm{IL}-2+\mathrm{S} / \mathrm{N} 1: 3\end{array}$ & $\begin{array}{l}\mathrm{HCl}- \\
\text { Normal } \\
\text { Normal } \\
\text { IL-2 }\end{array}$ & $\begin{array}{rl} & 714 \\
7 & 224 \\
1 & 732(164) \\
892(143) \\
1394(189)\end{array}$ & $\begin{array}{r}15,6 \\
2,7 \\
10,4\end{array}$ & $\begin{array}{l}<0,01 \\
<0,001\end{array}$ \\
\hline $\begin{array}{c}\text { A } 11 \\
\text { (Cible ALLO) }\end{array}$ & $\begin{array}{c}0 \text { (Spont) } \\
0 \\
5: 1 \\
5: 1 \\
5: 1\end{array}$ & $\begin{array}{c}\overline{I L-2} \\
\mathrm{~S} / \mathrm{N} 1: 3 \\
\mathrm{IL}-2+\mathrm{S} / \mathrm{N} 1: 3\end{array}$ & $\begin{array}{c}\mathrm{HCl} \text { (Total) } \\
\text { Normal } \\
\text { Normal } \\
\text { IL-2 }\end{array}$ & $\begin{array}{l}2325 \\
7904 \\
6313(266) \\
4922(442) \\
5478(501)\end{array}$ & $\begin{array}{l}71 \\
47 \\
57\end{array}$ & $\begin{array}{l}<0,01 \\
N S\end{array}$ \\
\hline
\end{tabular}

( ) : SD

Le facteur inhibe la croissance (fig. 1) et semble agir comme s'il diminuait le nombre de récepteurs IL-2 accessibles de la lignée IL-2 dépendante de référence, CTLL-2 (fig. 2), mesurés à la trieuse automatique de cellules (FACS). Le matériel inhibiteur semble sortir vers 70-72 Kd en HPLC, et avoir un Pi d'environ 5.

Si on cultive $48 \mathrm{~h}$ des cellules humaines d'un donneur $A$ dans un milieu contrôle, ou dans du surnageant du choriocarcinome JEG fortement dilué ( $1: 8000)$, puis qu'on lave 3 fois ces cellules dans du milieu $(50 \mathrm{ml}$, pour éliminer

Reproduction, Nutrition, Développement, $\mathrm{n}^{\circ} 6 \mathrm{~B} / 88-5$ 


\section{SURNAGEANT 明瓶SURNAGEANT DILUE DANS L'INTERLEUKINE 2. \%d' Inhibition}

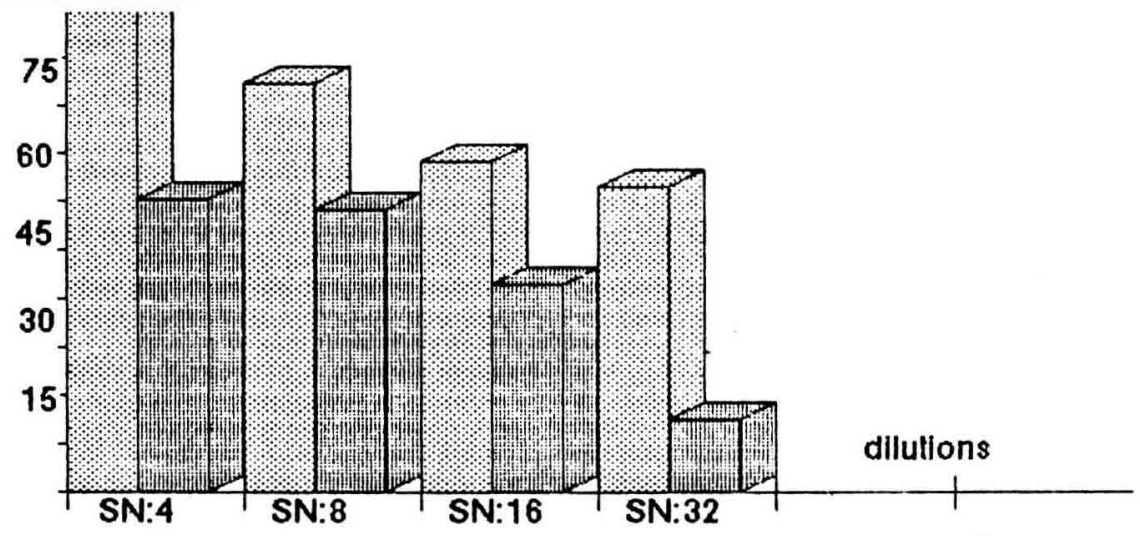

FIG. 1. - Inhibition de la croissance de la lignée CTLL-2 par le surnageant de cultures de placenta humain. Abolition de cette inhibition par culture en présence d'un excès d'Interleukine 2.

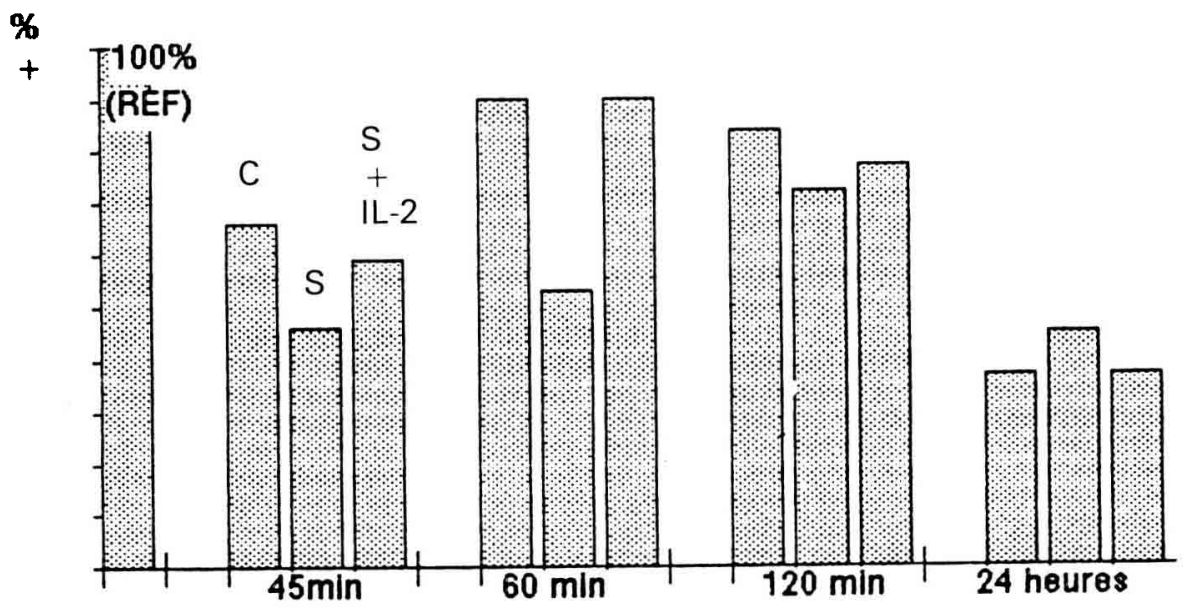

FIG. 2. - Inhibition en une culture de CTLL -2 en présence de surnageant de l'expression du récepteur IL-2 haute affinité marqué en fluorescence indirecte par l'anticorps monoclona/5A2 (fourni par J. Theze et J. L. Moreau, Institut Pasteur). La mesure est effectuée à la trieuse automatique de cellules (FACS). La 1 ère colonne représente un $100 \%$ de positif de référence pour le graphique.

Par la suite, les colonnes représentent à $45 \mathrm{~min}$ de culture, $60 \mathrm{~min}, 120 \mathrm{~min}$ et $24 \mathrm{~h}$ l'expression du R IL-2 en présence, à la sortie d'une expansion optimale, de milieu contrôle, de surnageant à une dose fixe $(1: 4)$, ou du même surnageant à la même dose, mais dans une dose d'Interleukine 2. La décroissance des cellules positives à $24 \mathrm{~h}$ est liée au cycle d'activation de la cellule. On notera que tout se passe comme si, à $24 \mathrm{~h}$, après consommation du matériel inhibiteur, les cellules rentraient en cycle et exprimaient alors plus de R IL-2 que les contrôles déjà en cycle depuis 24 h.

toute possibilité de transport de matériel inhibiteur, puisque le S/N de JEG n'est plus inhibiteur en test direct au-delà du $20000^{\circ}$ ) et qu'on réalise alors une réaction lymphocytaire mixte anti-cellules $B$ en présence de cellules $A$ ainsi 
"éduquées》 ou pseudo-éduquées, irradiées $1500 \mathrm{R}$ comme les cellules B stimulatrices, on observe une très forte inhibition de la MLC. Le surnageant a donc induit de (très) fortes cellules suppressives (tabl. 2). L'effet de ces facteurs se complète par l'action d'hormones. Ainsi nous avons montré que la progestérone faisait libérer aux lymphocytes CD8 + un facteur immunosuppressif de $34 \mathrm{Kd}$, pourvu qu'il s'agisse de lymphocytes de femme enceinte (Szekeres Bartho et al., 1988). Ceci pose le problème du mécanisme «activateur 》 des CD8+ pendant la gestation en préalable à l'action de la progestérone, et de l'existence d'un récepteur spécifique pour la progestérone sur de tels lymphocytes (puisque le RU 4386 bloque l'action du facteur). Plus récemment, il a été montré dans le laboratoire que le 1-25(OH) 2 D3, métabolite de la vitamine $\mathrm{D}$, produit en grande quantité par le placenta, inhibe une réaction lymphocytaire mixte et une lyse NK (fig. 3). Le mécanisme de ces inhibitions est à présent à l'étude dans le laboratoire.

\section{TABLEAU 2}

Induction de cellules suppressives de la réaction lymphocytaire mixte par le surnageant du choriocarcinome JEG.

\begin{tabular}{|c|c|}
\hline MLC en présence de & Cpms \\
\hline \multirow{8}{*}{$\begin{array}{l}\text { Milieu de culture contrôle } \\
\text { Cellules pseudo-éduquées } \\
\text { Cellules éduquées en S/N de JEG } 1: 2 \\
1: 500 \\
1: 1000 \\
1: 2000 \\
1: 4000 \\
1: 8000\end{array}$} & 89946 \\
\hline & 86406 \\
\hline & 3433 \\
\hline & 2332 \\
\hline & 1504 \\
\hline & 1924 \\
\hline & 6394 \\
\hline & 5704 \\
\hline
\end{tabular}

L'éducation des cellules est réalisée par culture de lymphocytes périphériques du même donneur que les cellules répondeuses de la réaction lymphocytaire mixte pendant $48 \mathrm{~h}$ dans du milieu contrôle ou contenant diverses dilutions de surnageant de choriocarcinome JEG. Les cellules ainsi " éduquées " sont irradiées 1500 rads, et rajoutées dans un volume $1: 1: 1$ répondeurs : cellules éduquées stimulatrices à une MLC qui est récoltée 5 jours après (MLC humaine en $10 \%$ SVF).

Mais le placenta entretient aussi un dialogue "positif » avec le système immunitaire maternel. Ainsi, les facteurs placentaires ne bloquent que certaines fonctions des cellules T. La sécrétion des CSFs (Colony stimulating factor) n'est pas touchée. Or, in vitro ces facteurs sont des facteurs de croissance pour le trophoblaste et les macrophages placentaires (Athanassakis et al., 1987 ; Armstrong et Chaouat, 1987). De fait, les placentas de gestation allogénique sont plus gros que les gestations isogéniques (Hamilton et Hamilton, 1987b) et (T. G. Wegmann, communication personnelle), il y a plus de GM-CSF (CSF Beta un, granulocyte macrophage colony stimulating factor) dans une déciduale de gestation syngénique qu'allogénique, et plus d'activité HCSF (histamine colony stimulating factor qui est en fait le GM-CSF), selon des expériences récentes que nous menons avec Michel Dy et Marianne Minkowski (U 25 INSERM), dans une déciduale $\mathrm{CBAJ} \times \mathrm{Balb} / \mathrm{c}$ que $\mathrm{CBA} / \mathrm{J} \times \mathrm{DBA} / 2$. 


\begin{tabular}{|c|c|}
\hline control & 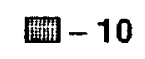 \\
\hline
\end{tabular}

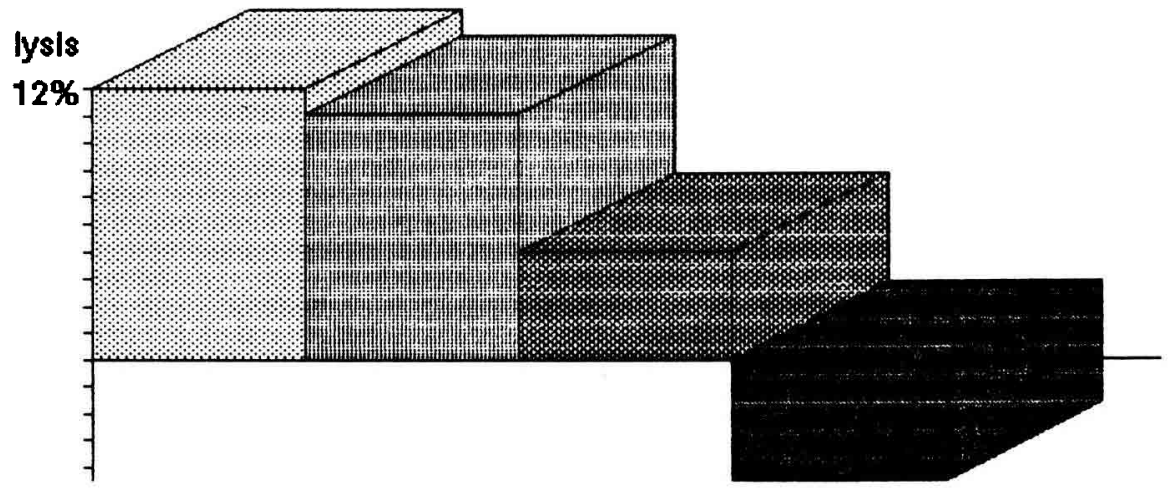

FIG. 3. - Inhibition par des concentrations décroissantes de 1-25 $(\mathrm{OH})_{2} \mathrm{D} 3$ de la lyse NK sur la cible $K 562$. Les résultats sont exprimés en lyse spécifique, ce qui explique la «lyse négative » observée à $10-8 \mathrm{M}$.

Or nous disposons justement avec ces souches d'un modèle d'avortement murin d'origine immunitaire (Chaouat, Kiger et Wegmann, 1983) : la souris CBA a 30 à $40 \%$ de résorptions lorsqu'accouplée à $\mathrm{DBA} / 2$ et 5-15\% lorsqu'accouplée à Balb/c. Le fait important est que, dans ce système l'immunisation anti-Balb/c peut ramener le taux de résorptions de la combinaison $\mathrm{CBA} \times \mathrm{DBA} / 2$ à la normale (Chaouat et al., 1985 ; Kiger et al., 1985 ; Clark et al., 1986). Surtout l'effet protecteur peut être transféré par des cellules d'un animal préimmunisé à une $\mathrm{CBA} / \mathrm{J}$ vierge. Fait capital, le poids des unités fœto-placentaires des souris traitées revient à la normale (il y a hypotrophie des $C B A \times D B A / 2$ par rapport à $\mathrm{CBA} \times \mathrm{CBA}, \mathrm{CBA} \times \mathrm{Balb} / \mathrm{c})$. De même, dans le système de grossesses inter espèces embryon Ane $\times$ Ane transféré dans une jument, on observe un taux élevé (>90\%) d'échecs de la gestation. Or, fait intéressant, il y a défaut de croissance des villosités choriales qui vont donner naissance à la coupe endométriale (Antczack et Allen, 1984). Or l'immunisation active ou passive permet à ces embryons d'âne de " tenir », et ce par développement des dites villosités (Allen et al., 1986, 1987). Ces résultats suggèrent une action « immunotrophique », directe ou non, des lymphokines sur le placenta (Wegmann, 1984, 1988 ; Chaouat et al., 1987). Ceci a pu être démontré de diverses façons :

1) Une administration conjuguée de monoclonaux anti L3T4 et anti Lyt 2 empêche l'effet anti-avortif de l'immunisation CBA anti Balb/c et augmente le taux d'avortement de CBA $\times$ Balb/c au niveau de CBA $\times$ DBA/2 (Chaouat et al., 1988) (tabl. 3). On notera les 2 importantes corrélations que sont les poids placentaire et la phagocytose de billes de latex, mesurée selon Athanassakis (Athanassakis et al., 1987).

2) Les souris auto-immunes $M R L / p r / I p r$ ont une nette hypertrophie placentaire. Celle-ci peut être corrigée par injection des mêmes monoclonaux (tabl. 4). Or les cellules T de ces souris secrètent anormalement trop d'IL-3 (CSF bêta 2), et des anticorps "IL-3 like" (Weston et al., 1987; Ohta et al., 1988). 


\section{TABLEAU 3}

Abolition de l'effet anti-abortif de l'immunisation anti-Ba/b/C chez des femelles CBA/J par traitement de ces dernières par des injections d'anticorps monoclonaux anti L3T4 + anti LyT 2.1 purifiés par HPLC et injectés i.p. à la souris CBA/J aux jours 8, 10, 12 de gestation.

\begin{tabular}{|c|c|c|c|c|c|}
\hline \multirow[b]{2}{*}{ Souche } & \multicolumn{2}{|c|}{ Traitement } & \multirow[b]{2}{*}{ Résorptions } & \multirow[b]{2}{*}{ Poids plac. } & \multirow[b]{2}{*}{ Phagocytose plac } \\
\hline & 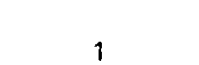 & 2 & & & \\
\hline $\begin{array}{l}\mathrm{CBA} \times \mathrm{DBA} / 2 \\
\mathrm{CBA} \times \mathrm{DBA} / 2 \\
\mathrm{CBA} \times \mathrm{DBA} / 2 \\
\mathrm{CBA} \times \mathrm{DBA} / 2\end{array}$ & $\begin{array}{l}\text { Imm a Balb/c } \\
\text { Imm a Balb/c } \\
\text { Imm a Balb/c }\end{array}$ & $\begin{array}{c}- \\
\overline{A c C t I} \\
\mathrm{AcMcl}\end{array}$ & $\begin{aligned} 42,1 \% & <0,001 \\
9,9 \% & \\
6,3 \% & <0,001 \\
51,3 \% & <0\end{aligned}$ & $\begin{array}{r}67,3 \mathrm{mg}(13,9) \\
124,8 \mathrm{mg}(17,9)<0,001 \\
118,7 \mathrm{mg}(12,5) \\
62,4 \mathrm{mg}(19,9)\end{array}$ & $\begin{array}{l}185 U \\
274 U \\
246 U \\
162 U\end{array}$ \\
\hline
\end{tabular}

AcCtl : Traitement par des lgGs de rat à la même concentration que le cocktail d'anticorps monoclonaux $(\mathrm{AcMcl})$ de rat utilisés. Ce cocktail est de même isotype cytotoxique que les anticorps expérimentaux.

Imm a Balb/c : Préimmunisation avant l'accouplement à DBA/2 des CBA/J contre des lymphocytes Balb/c.

(): SD.

\section{TABLEAU 4}

Correction de l'hypertrophie placentaire chez Mrl lpr/lpr par injection d'anti L3T4 (GK.15) + anti LYT 2.1 purifiés par HPLC. Le poids et l'activité phagocytaire placentaire sont mesurés au jour 14 ou 15.

\begin{tabular}{|c|c|c|c|c|c|}
\hline \multirow{2}{*}{$\begin{array}{l}\text { Souche } \\
\text { MRL normale } \\
\text { MRL normale }\end{array}$} & \multirow{2}{*}{$\begin{array}{c}\text { Traitement } \\
\text { IgG contrôles } \\
\text { Monoclonaux }\end{array}$} & \multicolumn{2}{|c|}{ Poids plac. moyen } & \multicolumn{2}{|c|}{ Indice phag. moyen } \\
\hline & & $\begin{array}{l}133,6 \mathrm{mg}(18,6) \\
103,3 \mathrm{mg}(16,5)\end{array}$ & $<0,001$ & $\begin{array}{l}76,1 \cup(45) \\
71,3 \cup(28)\end{array}$ & NS \\
\hline $\begin{array}{l}\text { MRL Mp Ipr//pr } \\
\text { MR Mp Ipr/Ipr }\end{array}$ & $\begin{array}{l}\text { IgG contrôles } \\
\text { Monoclonaux }\end{array}$ & $\begin{array}{r}166,2 \mathrm{mg} \\
93,7 \mathrm{mg}\end{array}$ & $<0,001$ & $\begin{array}{l}97,6 \cup(41) \\
73,3 \cup(44)\end{array}$ & $<0,01$ \\
\hline $\begin{array}{r}\text { C3H (Contrôles) } \\
\text { (Pesée }\end{array}$ & arée) ${ }^{\text {Nil }}$ & $109,3 \mathrm{mg}$ & & & \\
\hline
\end{tabular}

() : SD

3) Surtout, on peut prévenir les avortements chez CBA $\times \mathrm{DBA} / 2$ en transférant des cellules de MRL Ipr/lpr, qui n'ont jamais vu les antigènes $\mathrm{H}-2 \mathrm{~d}$ de DBA/2 (ou de $\mathrm{Balb} / \mathrm{c}$ ) - bien que le transfert de ces cellules ait à terme des inconvénients pour la souris CBA/J - H-2k comme MRL Ipr/Ipr, - savoir des syndromes auto-immuns, intestinaux surtout (tabl. 5).

En fait, cette manipulation suggérait que les lymphokines pouvaient agir par elles-mêmes, l'immunisation réalisant le " priming », et, par son côté spécifique, forçant lors du trafic cellulaire le bon "targeting » antigénique.

De fait, on peut diminuer de façon très significative les résorptions par du $\mathrm{GM}$ CSF naturel (P 338 D1) ou recombinant (Genzyme) (Chaouat et al., 1988; D. A. Clark, communication personnelle confirmative; Chaouat, Clark, Wegmann et al., soumis), et l'IL-3 (tabl. 6). 
TABLEAU 5

Correction du taux d'avortement dans la combinaison CBA $\times D B A / 2$ par injection de lymphocytes de souris auto-immunes MRL /pr//pr, mais non par injection de cel/ules de souris MRL/L normales. Le traitement des animaux aux jours 8, 10 et 12 par les anticorps monoclonaux ci-dessus décrits empêche l'effet du transfert.

\begin{tabular}{|c|c|c|c|c|}
\hline Souris & $\operatorname{Tt} n^{\circ} 1$ & $\operatorname{Tt} n^{\circ} 2$ & Résorptions & Poids placentaire \\
\hline $\mathrm{CBA} / \mathrm{J} \times \mathrm{Balb} / \mathrm{c}$ & - & - & $8,9 \%$ & $137,9(25,7)$ \\
\hline $\mathrm{CBA} / \mathrm{J} \times \mathrm{Balb} / \mathrm{c}$ & MRL NI & - & $7,8 \%$ & $142,7(14,8)$ \\
\hline $\mathrm{CBA} / \mathrm{J} \times \mathrm{Balb} / \mathrm{c}$ & $\mathrm{MRL} \mathrm{Ipr} / \mathrm{lpr}$ & & $6,3 \%$ & $165,4(19,7)$ \\
\hline $\mathrm{CBA} / \mathrm{J} \times \mathrm{Balb} / \mathrm{c}$ & - & Ac Ctrôle & $9,7 \%$ & $145,3(16,5)$ \\
\hline $\mathrm{CBA} / \mathrm{J} \times \mathrm{Balb} / \mathrm{c}$ & MRL NI & Ac Ctrôle & \multirow{2}{*}{\multicolumn{2}{|c|}{ non fait }} \\
\hline $\mathrm{CBA} / \mathrm{J} \times \mathrm{Balb} / \mathrm{c}$ & MRL NI & $\mathrm{A} \operatorname{CD} 4+\mathrm{CD} 8$ & & \\
\hline $\mathrm{CBA} / \mathrm{J} \times \mathrm{Balb} / \mathrm{c}$ & MRL Ipr.Ipr & Ac Ctrôle & $6,9 \%$ & $170,9(23,7)$ \\
\hline $\mathrm{CBA} / \mathrm{J} \times \mathrm{Balb} / \mathrm{c}$ & - & $A \mathrm{CD} 4+\mathrm{CD} 8$ & $38,9 \%$ & $93,2(18,1)$ \\
\hline $\mathrm{CBA} / \mathrm{J} \times \mathrm{DBA} / 2$ & - & Ac Ctrôle & $41,7 \%$ & $85,7(14,8)$ \\
\hline $\mathrm{CBA} / \mathrm{J} \times \mathrm{DBA} / 2$ & Balb/c & - & $10,2 \%$ & $146,2(18,3)$ \\
\hline $\mathrm{CBA} / \mathrm{J} \times \mathrm{DBA} / 2$ & MRL NI & - & $33,1 \%$ & $101,6(14,1)$ \\
\hline $\mathrm{CBA} / \mathrm{J} \times \mathrm{DBA} / 2$ & $\mathrm{MRL} / \mathrm{pr} / \mathrm{lpr}$ & - & $13,7 \%$ & $172,4(22,9)$ \\
\hline $\mathrm{CBA} / \mathrm{J} \times \mathrm{DBA} / 2$ & $\mathrm{MRL} / \mathrm{pr} / \mathrm{lpr}$ & Ac Ctrôle & $15,5 \%$ & $168,9(19,6)$ \\
\hline $\mathrm{CBA} / J \times \mathrm{DBA} / 2$ & MRL Ipr//pr & $\mathrm{A} C D 4+\mathrm{CD} 8$ & $28,4 \%$ & $110,6(17,9)$ \\
\hline
\end{tabular}

Traitement $\mathrm{n}^{\circ} 1$ : cellules transférées au jour 0 .

Traitement $n^{\circ} 2$ : anticorps injectés aux jours 8,10 et 12 : anticorps contrôle (IgGs de rat). ou anti CD4 (anti L3T4, hyb GK 15) plus anti CD8 (Hyb 129).

Résorptions comptées au jour 14. Poids placentaire en mg.

TABLEAU 6

Traitement des résorptions $C B A \times D B A / 2$ par le GM-CSF et /'/L-3.

Souches $\quad \%$ Résorptions (CBA/J âgées de 3 mois)

\begin{tabular}{|c|c|}
\hline xpérience 1 & \\
\hline $\mathrm{CBA} \times \mathrm{DBA} / 2$ & 66 \\
\hline $\begin{array}{l}\mathrm{CBA} \times \mathrm{Balb} / \mathrm{c} \\
\mathrm{CBA} \times \mathrm{DBA} / 2\end{array}$ & 17,5 \\
\hline$+\mathrm{GM}-\mathrm{CSF} J 8,10,12$ & 10 \\
\hline
\end{tabular}

Expérience 2 :

\begin{tabular}{|c|c|c|c|}
\hline $\mathrm{CBA} \times \mathrm{DBA} / 2$ & $51,7 \%$ & Poids plac. moyen & $102,3 \mathrm{mg}$ \\
\hline $\mathrm{CBA} \times \mathrm{Balb} / \mathrm{C}$ & $17,2 \%$ & Poids plac. moyen & $141,7<0,01$ \\
\hline $\begin{array}{l}+ \text { GM-CSF J } 8,10,12 \\
+ \text { IL-3 de WEHI } \\
+ \text { R GMCSF }\end{array}$ & $\begin{array}{l}24 \% \\
28 \% \\
29,78 \%\end{array}$ & $\begin{array}{l}\text { Poids plac. moyen } \\
\text { Limite }(0,05) \\
\text { Poids plac. moyen }\end{array}$ & $\begin{aligned} 139,7 & <0,001 \\
\text { nonpesé } & \\
132,6 & <0,001\end{aligned}$ \\
\hline
\end{tabular}

Le GM-CSF est du GM CSF obtenu par purification de surnageant de culture en sérum humain $A B$ de la tumeur P 338 D1. (II est fourni par Michel Dy et Marianne Minkowski, U 25 INSERM). L'IL-3, aussi fourni par eux, vient de la tumeur WEHI. Le GM-CSF recombinant murin est de source commerciale (GENZYME). 
A l'inverse, on peut augmenter le taux d'avortements par les lymphokines suivantes : TNF alpha (même humain) (tabl. 7). L'interféron gamma recombinant à hautes doses, semble-t-il (expériences confirmatives en cours) fait de même et (court-circuitant les facteurs placentaires?) I'IL-2 (Tezabwala et Johnstone, 1986 ; Panayi, communication orale à la British Society for Immunology, 1983 ; P. Johnson, communication personnelle ; Scodras et Lala, 1987 ; Chaouat, 1989 , sous presse et en préparation). On peut enfin le faire par des NKs activées par le poly (I) poly (C) ou le poly (1) (C12) U (Chaouat et al., en préparation ; Chaouat et al., 1988 et en préparation, De Fougerolles et Baines, 1987) (tabl. 8).

TABLEAU 7

Augmentation des résorptions $C B A \times D B A / 2$ par injection de TNF ALPHA recombinant humain.

$\mathrm{CBA} \times \mathrm{DBA} / 2$, Injectées avec P.B.S.

$42,8 \% \mathrm{R} /(\mathrm{R}+\mathrm{F})$

$\mathrm{CBA} \times \mathrm{DBA} / 2$, Injectées TNF Recombinant

$78,6 \%$

$<0,01$

Le TNF recombinant alpha est un cadeau du Dr J. Wietzerbin (U 196 INSERM).

TABLEAU 8

Augmentation des résorptions $C B A \times D B A / 2$ par Poly (I) (C).

$\begin{array}{lrr}\mathrm{CBA} \times \mathrm{DBA} / 2 & 37,8 \% & <0,02 \\ \mathrm{CBA} \times \mathrm{Balb} / \mathrm{c} & 9,6 \% & \\ \mathrm{CBA} \times \mathrm{DBA} / 2 & 57,6 \% & <0,05 \\ \mathrm{POLY}(\mathrm{I})(\mathrm{C}) \mathrm{J} 7 & 76,3 \% & <0,01 \\ \mathrm{POLY}(\mathrm{I})(\mathrm{C} 12 \mathrm{U}) \mathrm{J} 7 & & \end{array}$

Le Poly (I) (C) et le Poly (I) (C12U) ont été fournis par le Dr M. N. Thang (U 249 INSERM).

Or, de tels mécanismes ou lymphokines sont en jeu s'il existe une infection locale. On voit donc se profiler une explication "immuno-infectieuse" des avortements répétitifs (Hamilton et Hamilton, 1987a), à côté de l'explication purement immunotrophique de leur traitement.

Ceci nous laisse entrevoir l'extrême beauté des mécanismes régulateurs qui seront, à n'en pas douter, un sujet d'étude passionnant de la prochaine décade de l'immunologie de la reproduction, avec des applications thérapeutiques et vétérinaires ( $v$ compris industrielles) non négligeables : sans doute saurons-nous assez vite le mécanisme de l'immunisation anti-lymphocytes chez l'homme (voir 18th RCOG study group) et y aura-t-il des applications très rapides chez certains animaux: c'est déjà le cas pour accroître la prolificité des porcs (Stone, communication orale au Faseb Meeting 1987) et les enjeux économiques dans ce dernier secteur ne sont pas du tout négligeables à en croire Canadiens et Australiens. 
Addendum. - Juste après la rédaction de ce manuscrit (en août 1988), Julia SzekeresBartho, en coopération avec Georges Szekeres, Hôpital de l'Hôtel-Dieu, a montré par immuno-histo-chimie dans notre laboratoire que le monoclonal Mpri anti-récepteur de progestérone de souris marquait exclusivement les lymphocytes CD8 + de femme enceinte, et non les lymphocytes CD4 de ces dernières, ni les CD8 des femmes hors gestation. Ce récepteur est inductible par stimulation allogénique ou par la PHA (phyto-haemaglutinnine).

\section{Références}

ALLEN W. R., KYDD J. H., ANTCZACK D. F., 1986. Successful application of immunotherapy to a model of pregnancy failure in equids. In D. A. CLARK, B. A. CROY, Reproductive immunology. Elsevier.

ALLEN W. R., KYDD J., DONALDSON W. L., ORIOL J. G., ANTCZAK D., 1987. Expression of immune response to fetal antigens in equine pregnancy, p. 255. In Coll. INSERM CNRS Immunologie de la reproduction : Relation materno fetale. G. CHAOUAT Ed. Coll., 154. Ed. INSERM, p. 255.

ANTCZACK D. F., ALLEN W. R., 1984. Invasive trophoblast in the genus equus. Ann. immunol. (Inst. Pasteur), $135 \mathrm{D}, 325-331$.

ARMSTRONG D. A., CHAOUAT G., 1987. Effects of lymphokines and immune complexes on murine placental growth in vitro. Human reproduction Meet., Tokyo (et soumis par publication).

ATHANASSAKIS I., BLEACKLEY R. C., PAETKAU V., GUILBERT L., BARR P. J., WEGMANN T. G., 1987. The immunostimulatory effects of $T$ cells and $T$ cell lymphokines on murine fetally derived placental cells. J. Immunol., 138, 37-44.

BELL S. C., BILLINGTON W. D., 1983. Antifetal alloantibody in the pregnant female. Immunol. Rev., 75, 5-31.

BILLINGTON W. D., BURROWS F. J., 1986. The rat placenta expresses paternal class I MHC antigens. J. Reprod. Immunol., 9, 155-160.

BULMER J. D., RITSON A., 1988. The decidua in early pregnancy, 171-181. In Eariy pregnancy loss, mechanisms and treatment. 18th RCOG Study group, Roy. Coll. Obstet. Gynaecol. London.

CHAOUAT G., VOISIN G. A., ESCALIER D., ROBERT P., 1979. Facilitation reaction (enhancing antibodies and suppressor cells) and rejection reaction (sensitised cells) from the mother to the paternal antigens of the conceptus. Clin. exp. Immunol., 35, 13-24.

CHAOUAT G., DUCHET SUCHAUX M., CHAFFAUX S., VOISIN G. A., 1980. Immunoactive products of the placenta: I) Immunosuppressive properties of crude and water soluble extracts. J. Reprod. Immunol., 2, 121-128.

CHAOUAT G., FOWLKES B. J., LEISERSON W., ASOFSKY R. A., 1982. Modifications of the thymocytes subsets during pregnancy analysed by flow microfluorometry: Role of the alloantigenic status of the conceptus. Thymus, 4, 229-238.

CHAOUAT G., KIGER N., WEGMANN T. G., 1983. Vaccination against spontaneous abortion in mice. J. Reprod. Immunol., 5, 389-394.

CHAOUAT G., MONNOT P., 1984. Systemic active suppression is not necessary for successful allopregnancy. Amer. J. Reprod. Immunol., 6, 5-8.

CHAOUAT G., KOLB J. P., KIGER N., STANISLAWSKI M., WEGMANN T. G., 1985. Immunological concommitants of vaccination against abortion in mice. J. Immunol., 134, 1594-1602.

CHAOUAT G., KOLB J. P., 1985 . Immunoactive products of placenta. IV. Impairment by placental cells and their products of CTL function at the effector stage. J. Immunol., 135, 215-222.

CHAOUAT G., KOLB J. P., CHAFFAUX S., RIVIĖRE M., ATHANASSAKIS I., GREEN D., WEGMANN

T. G., 1986. The placenta and the survival of the fetal allograft. In T. G. WEGMANN,

T. J. GILL III, Reproductive immunology II. Oxford Univ. Press.

CHAOUAT G., 1987. Placental immunoregulatory factors. J. Reprod. Immunol,, 10, 179-188 
CHAOUAT G., LANKAR D., KOLB J. P., CLARK D. A., 1987. Deux modèles d'avortements d'origine immunitaire chez la souris de laboratoire : mécanismes abortifs, modalités et mécanismes du traitement par l'immunisation contre un mâle relié ou non relié suivant les différences antigéniques père-mère, p. 243. In Coll. INSERM CNRS /mmunologie de la relation feto-materne/le. G. CHAOUAT Ed. Editions INSERM, J. LIBBEY. Vol. 154, p. 243.

CHAOUAT G., MENU E., SADELAIN M., ATHANASSAKIS I., WEGMANN T. G., 1988a. Placental immunotrophism : maternal T cells contribute to the growth and survival of the fetal allograft. Amer. J. Reprod. Immunol., 16, 70.

CHAOUAT G., CLARK D. A., WEGMANN T. G., 1988b. Genetic aspects of the CBA $\times$ DBA $/ 2$ and B10 $\times$ B10. A models of murine pregnancy failure and its prevention by lymphocyte immunisation, 89-103. In R. W. BEARD et F. SHARP Early pregnancy loss, mechanisms and treatment. 18th RCOG Study group, Roy. Coll. Obstet. Gyanaecol. London, 89-103

CHAOUAT G., 1988. Sta-Put analysis of $\mathrm{CBA} \times \mathrm{DBA} / 2$ vs $\mathrm{CBA} \times \mathrm{Balb} / \mathrm{c}$ or CBA (anti $\mathrm{Balb} / \mathrm{c}) \times \mathrm{DBA} / 2$ murine placenta: relative deficiency of cellular inhibitors of cellular immune responses. Amer. J. Reprod. Immunol., 16, 70.

CHAVEZ D. J., McINTYRE J. A., COlliver J. A., PAGE FAULK W. P., 1987. Allogeneic matings and immunisation have different effects on nulliparous and multiparous mice. $J$. Immuno/., 139, 85-88.

CLARK D. A., SLAPSYS R. M., CROY B. A., ROSSANT J., 1984. Immunoregulation of host versus graft responses in the uterus. Immunol. Today, 5, 11-13

CLARK D. A., 1985. Maternal Immune response to the fetus. EOS-Rev. Immunol. Immunofarmacol., 2. 114-117.

CLARK D. A., CHAOUAT G., 1986. Characterisation of the cellular basis for the inhibition of cytolytic effector cells by the murine placenta. Cell. Immunol., 102, 43-51.

CLARK D. A., CHAPUT A., TUTTON B., 1986. Active suppression of host versus graft reaction in pregnant mice. VII. Spontaneous abortion of CBA $\times$ DBA/2 fetuses in the uterus of $\mathrm{CBA} / \mathrm{J}$ mice correlates with deficient non-T suppressor cell activity. J. Immunol., 136, 16-68.

CLARK D. A., DAMJI N., CHAPUT A., DAYA S., ROSENTHAL K. L., BRIERLEY J., 1987. Decidua associated suppressor cells and suppressor factors regulating interleukin $2:$ their role in the survival of the «fetal allograft». In B. CINADER, R. G. MILLER, Prog. Immunol, Acad. Press, New York, 6, 1089-1099.

CROY B. A., ROSSANT J., 1987. Mouse embryonic cells become susceptible to CTL mediated lysis after midgestation. Cell. /mmunol., 104, 355-365.

DE FOUGEROLLES R., BAINES M., 1987. Modulation of Natural Killer activity influences resorbtion rates in CBA $\times$ DBA/2 matings. J. Reprod. Immunol., 11, 147-153.

DORMAN P. J., SEARLE R. F., 1988. Alloantigen presenting capacity of human decidual tissue. $J$. Reprod. Immunol., 13, $101-113$.

DRAKE B. L., HEAD J. R., 1988a. Murine trophoblast cells are susceptible to Lymphokine Activated killer (LAK) cell lysis. Amer. J. Reprod. Immunol., 16. 114.

DRAKE B. L., HEAD J. R., HEDLEY M. L., TUCKER P., FORMAN J., 1988b. Ontogeny of class I MHC gene expression in the mouse fetus and placenta. Amer J. Reprod. Immuno/., 16, 111.

DUC H. T., MASSE A., BOBE P., KINSKY R. G., VOISIN G. A., 1985. Deviation of humoral and cellular alloimmune reactions by placental extracts. J. Reprod. Immunol., 7, 27-39.

HAMILTON M. S., HAMILTON B. L., 1987a. Environmental influences on immunologically associated recurent spontaneous abortion in CBA/J mice. J. Reprod. Immuno/., 16. 153-155.

HAMILTON B. S., HAMILTON M., 1987b. Effect of maternal-fetal histoincompatibility on the weight of the feto placentalunit in mice : the role of minor histocompatibility antigens. Amer. J. Reprod. Immunol., 4, 153-156.

HUNT J. S., FISHBACK J. L., 1988. Class I antigen expression by human extraembryonic cells. Amer. J. Reprod. Immunol., 16, 111

KIGER N., CHAOUAT G., KOLB J. P., WEGMANN T. G., GUENNET J. L., 1985. Immunogenetic studies of spontaneous abortion in mice : I) Preimmunisation of the mother with allogeneic spleen cells. J. Immunol., 134, $2966-2972$.

KOLB J. P., CHAOUAT G., 1984. Immunoactive products of the placenta: III) Suppression of natural killing activity. J. Immuno/., 132, 2305-2310. 
LANMAN J. T., DINENSTEIN J., FIKRING S., 1962. Homograft immunity in pregnancy. Lack of harm to fetus from sensitization of the mother. Ann. N. Y. Acad. Sci., 99, 706-708.

MATTHEWS C. J., SEARLE R. F., 1988. Antigen presenting capacity of murine decidual cells in vivo. J. Reprod. Immunol., 12, 4, 287-297.

MATTSON R., MATTSON A., SULILA P., 1985. Allogeneic pregnancy in B cell depleted CBA/Ca mice effects on fetal survival and maternal lymphoid organs. Dev. comp. Immunol., 9, $709-714$.

MATTSON R., HOLMDAL R., 1987. Maintained allopregnancy in rats depleted of cytotoxic/suppressor cells by 0X8 monoclonal antibody treatment. $J$. Reprod. Immunol., 12. 23-35.

MENU E., CHAOUAT G., 1988. Immunoregulatory molecules secreted by normal placenta and gestational tumors. In Immunology of the fetus. CRC Press. Boca raton. Florida. (In press.)

MITCHISON N. A., 1953. The effect on the offspring of maternal immunisation in mice. J. Genet., 5, 406-411.

OHTA Y., TAMURA S., TEZUKA E., SUGAWARA S., IMAI S., TANAKA H., 1988. Autoimmune MRL/Ipr sera contains IgG with Interleukin like activity. J. Immunol., 140, 520-525.

RODGER J. C., 1985. Lack of requirement for a maternal humoral immune response to establish or maintain a succesful allogeneic pregnancy. Transplantation, 40, 372-375.

SCODRAS J. M., LALA P. K., 1987. Reactivation of maternalleukocytes in the decidua with indomethacin, II-2 or combination therapy is associated with embryonic demise. Amer. J. Reprod. Immunol., 14, 12.

SLAPSYS R., BEESON J., CLARK D. A, 1986. Recruitment of a trophoblast dependent suppressor cell to the implantation site of allopregnant mice. Fed. Proceed., 44, 1880 (Abstr. 8582) et Tr. Doc. Etat Canadien Univ. McMaster, Ontario, Canada, 1987.

SMITH G., 1983. In vitro susceptibility of mouse placental trophoblast to cytotoxic effector cells. $J$. Reprod. Immunol., 5, 39-47.

SMITH R. N., POWELL A. E., 1977. The adoptive transfer of pregnancy induced hyporesponsiveness to male skin grafts by thymus dependent cells. J. exp. Med., 146, 899-905.

SUGAWARA M., HATTORI C., TEZUKA T., TAMURA S., OHTA Y., 1988. Monoclonal antibodies with Interleukin 3 activity derived from a MRL/Ipr mouse. J. Immunol., 140, 526-530.

SZEKERES BARTHO J., AUTRAND B., DEBRE P., ANDREU G., DENVER L., BLOT P., CHAOUAT G., 1988. Immunoregulatory effects of material secreted by healthy pregnant women lymphocytes after progesterone induction in vitro. $26^{e}$ Réun. Soc. franc. Etude Fertil., 1987. pp. $341-345$.

TARTAKOWSKY B., DE BAETSELIER P., FELDMAN M., SEGAL S., 1981. Sex-associated differences in the immune responses against fetal major histocompatibility antigens. Transplantation, 32, 395-397.

TEZABWALA B. U., JOHNSTONE P., 1986. Effects of administration of Interleukin 2 in pregnancy. J. Reprod. Immunol. Suppl. Juin 1986, p. 147

VOISIN G. A., CHAOUAT G., 1974. Demonstration, nature and properties of maternal antibodies fixed on placenta and directed against paternal alloantigens. J. Reprod. Fertil., Suppl. 21. 89-107.

WEGMANN T. G., WATERS C. A., DRELL D. W., CARLSON G. A., 1979. Pregnant mice are not primed but can be primed to fetal alloantigens. Proc. nat. Acad. Sci. USA, 76, 2410-2414.

WEGMANN T. G., 1984. Fetal protection against abortion : is it immunosuppression or immunostimulation? Ann. Immunol. Inst. Pasteur, 135 D, 309-311.

WEGMANN T. G., 1987. Placental Immunotrophism. Maternal T cells enhance placental growth and function. Amer. J. Reprod. Immunol., 15, 67-71.

WEGMANN T. G., 1988. Maternal T cells promote placental growth and prevent spontaeous abortion. Immunol. Letters, 17, 297-302.

WESTON K. M., YEH E. T. H., MAN-SUN SY, 1987. Autoreactivity accelerates the development of autoimmunity and lymphoproliferation in MRL/Mp lpr/lpr mice. J. Immunol., 139, 734-742.

ZUCKERMAN F. A., HEAD J. R., 1987. Murine trophoblast resist cell mediated lysis. 1) Resistance to allospecific cytotoxic T Iymphocytes. J. Immunol., 139, 2856-2865. 